\title{
Generalization of Shifted Fovea Multiresolution Geometries Applied to Object Detection
}

\author{
Fabián Arrebola, Pelegrín Camacho and Francisco Sandoval \\ Dpto. Tecnología Electrónica, E.T.S.I. Telecomunicación \\ Universidad de Málaga Campus de Teatinos, 29071 Málaga-SPAIN \\ E-mail: fabian@dte.uma.es
}

\begin{abstract}
This work describes a foveal vision system applied to object detection. The novelty of this system consists of carrying the detections using a generalization of the multiresolution shifted fovea images. The main advantage introduced is the great increase of the number of fovea positions allowed in shifted-fovea systems already implemented: this means that the maximum error of placement is reduced to one pixel, implying that any object could be examined at the highest resolution available regardiess of its coordinates. The concept is based on increasing the degrees of freedom and the related number of configuration parameters and the application of a new shifting algorithm which allows a higher number of fixation points on the scene and, therefore, reduces the error of fovea positioning on the region of interest and aproaches closer to the required scene details. Besides, we introduce the multiresolution data structure to manipulate and process this type of foveal geometries, as well as the results obtained after applying hierarchical algorithms for segmentation and detection of objects within this type of multiresolution images.
\end{abstract}

\section{Introduction}

Conventional vision systems operate with images of uniform resolution throughout the whole Field of Vision which, in many vision emulation tasks, implies huge resource usage or long processing times. Trying to improve any of those drawbacks, biological vision systems perform a sampling of the scene in a space-variant way which leads to the field of multiresolution vision systems. As one of them, Foveal Vision systems have among their main targets the performing of a selective reduction of data in order to shorten the required processing time and to achieve real time perception [1]. One of the basic tasks of active vision is to provide attention mechanisms to the vision system to detect and locate the most relevant details of the scene as well as gaze control capabilities to allow movement and fixation of fovea onto the regions of interest to let them be examined with the highest resolution available [2].

Eccentric or shifted-fovea geometries [3], have a cartesian topology with an exponentially decreasing resolution law, as proposed by Bandera and Scott [4] [5]. They are defined by four configuration parameters :

- $m$ : Number of rings or resolution levels surrounding the fovea.

- $d$ : Subdivision factor or number of subrings within each resolution ring.

$-s_{l}, s_{v}$ : Horizontal and vertical shifts applied to any ring $i$ within the ring $i+l$ which surrounds or contains ring $i$. They are given in rexels, or resolution cells of the rings.

The main features of these geometries are:

a) The relative shiftings, expressed in rexels, between succesive rings remain constant throughout the lattice, as shown in Fig 1.a. The associated hierarchical data structure named "assimetric foveal polygon -AFP-" has a regular and uniform connectivity law between the succesive resolution levels [9], as shown in Fig 1.b, which corresponds to the data mapping of the AFP into the image pyramid shown in Fig. 1.c. 


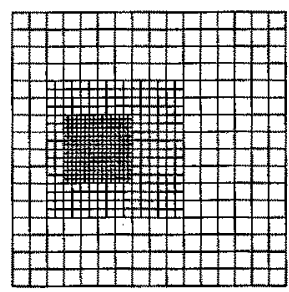

a

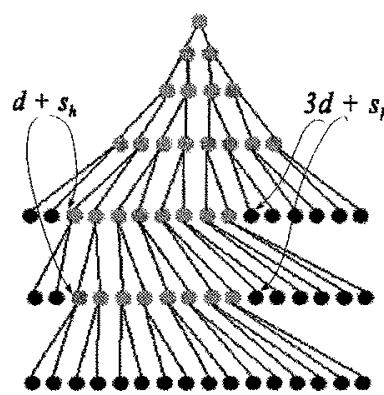

$b$

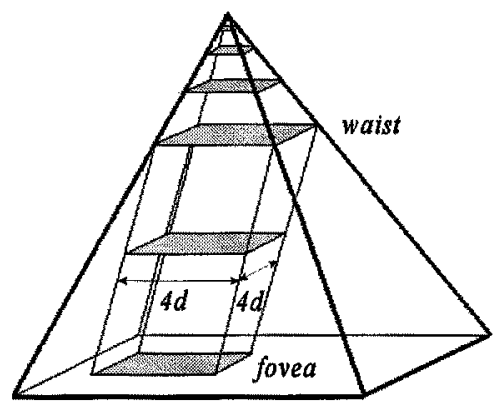

c

Fig 1. a) Shifted-fovea lattice $m=2, d=4, s_{h}=-2, s_{v}=0$. b) Unidimensional diagram of AFP. c) AFP data mapping into the image pyramid.

b) Since both $s_{\mathrm{h}}$ and $s$, may range between $-d$ and $d$, the number of possible geometries within the Field of Vision - FOV - for a given pair of $m$ and $d$ will be :

$$
N=(2 d+1)^{2}
$$

c) The minimum shift to be applied to any ring $i$ is related to the number of shiftable resolution levels in the lattice, having a value of :

$$
\Delta_{i}=2^{i+1}+2^{i+2}+\ldots+2^{m}=2^{m+1}-2^{i+1}
$$

which leads to the minimum shift that could be applied to the foveal region :

$$
\Delta_{\text {fovea }}=2^{1}+2^{2}+\cdots+2^{m}=2^{m+1}-2
$$

\section{Generalization of shifted-fovea geometries}

To obtain a generalization of the above geometries we assume that the shiftings within succesive resolution levels is not constrained to be a constant. This means that the number of the associated configuration parameters must be increased in order to specify for each ring or level its particular shift within the next higher level or containing ring. However, since for each level the shift is limited to the interval $[-d, d]$, in both vertical and horizontal directions, it implies that for any fovea position several lattices could exist, which requires a new shifting algorithm to relate just one lattice to each fovea position, or, in other words, to keep a single and unique hierarchical data structure.

The new set of configuration defining parameters are, besides $m$ and $d$, two arrays $S H$ and $S V$, each having $m-1$ elements, where each pair $\left(S H_{i}, S V_{i}\right)$ indicates the shifting in rexels of ring $i$ within ring $i+l$. The proposed shifting algorithm is an iterative one, having a worst case iteration number equal to $m$, by performing shifts of one resolution level per iteration. It starts by computing the shift of level $m-1$ :

$$
S H_{m-1}\left\{\begin{array} { l l } 
{ = - d \quad \text { if } ( \frac { S t _ { h } } { 2 ^ { m } } ) < - d } \\
{ = | \frac { S t _ { h } } { 2 ^ { m } } \rfloor \text { if } | \frac { S t _ { h } } { 2 ^ { m } } | < d } \\
{ = d \quad \text { if } ( \frac { S t _ { h } } { 2 ^ { m } } ) > d }
\end{array} \quad S V _ { m - 1 } \quad \left\{\begin{array}{ll}
=-d \quad \text { if }\left(\frac{S t_{v}}{2^{m}}\right)<-d \\
=\left|\frac{S t_{v}}{2^{m}}\right| \text { if }\left|\frac{S t_{v}}{2^{m}}\right|<d \\
=d \quad \text { if }\left(\frac{S t_{v}}{2^{m}}\right)>d
\end{array}\right.\right.
$$

where $\left(\mathrm{St}_{\mathrm{h}}, \mathrm{St}_{\mathrm{v}}\right)$ are the components of the total shifting vector to be applied to the fovea. 


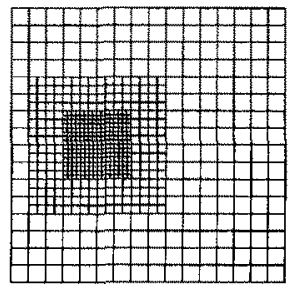

a

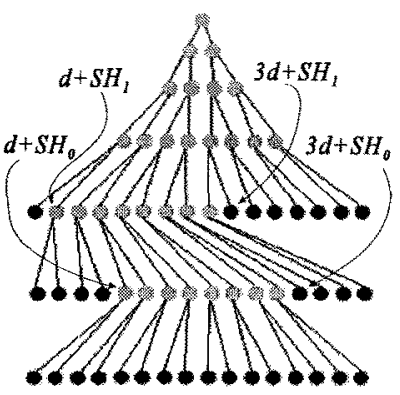

b

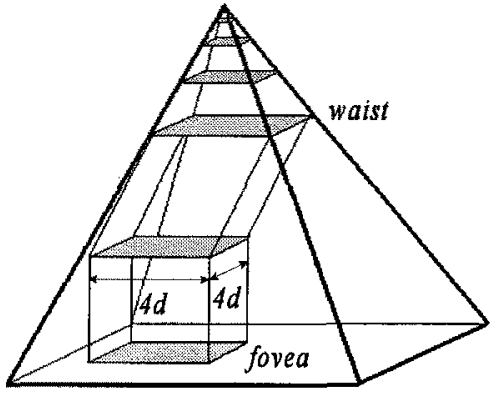

c

Fig 2. a) Lattice $m=2, d=4, S t_{h}=-12$ pixels, $S t_{v}=0$ pixels b) Unidimensional diagram of $A F P$. c) AFP data mapping into the image pyramid.

Before computing the shift corresponding to level $m-2,\left(\mathrm{St}_{\mathrm{h}}, \mathrm{St}_{\mathrm{v}}\right)$ must be updated by substracting to them the shift applied to its previous or upper level $m-1$ :

$$
\begin{aligned}
& S t_{h}:=S t_{h}-2^{m} \cdot S H_{m-1} \\
& S t_{V}:=S t_{v}-2^{m} \cdot S V_{m-1}
\end{aligned}
$$

therefore, the shift to apply to level $m-2$ will be :

$$
S H_{m-2}\left\{\begin{array} { l l } 
{ = - d \quad \text { if } ( \frac { S t _ { h } } { 2 ^ { m - 1 } } ) < - d } \\
{ = | \frac { S t _ { h } } { 2 ^ { m - 1 } } | \text { if } | \frac { S t _ { h } } { 2 ^ { m - 1 } } | < d } \\
{ = d \quad \text { if } ( \frac { S t _ { h } } { 2 ^ { m - 1 } } ) > d }
\end{array} \quad S V _ { m - 2 } \left\{\begin{array}{ll}
=-d & \text { if }\left(\frac{S t_{v}}{2^{m-1}}\right)<-d \\
=\left|\frac{S t_{v}}{2^{m-1}}\right| & \text { if }\left|\frac{S t_{v}}{2^{m-1}}\right|<d \\
=d & \text { if }\left(\frac{S t_{v}}{2^{m-1}}\right)>d
\end{array}\right.\right.
$$

being necessary to update $\mathrm{St}_{\mathrm{h}}$ and $\mathrm{St}_{y}$, substracting to their values the shift of the level $m-2$ :

$$
\begin{aligned}
& S t_{h}:=S t_{h}-2^{m-1} \cdot S H_{m-2} \\
& S t_{v}:=S t_{v}-2^{m-1} \cdot S V_{m-2}
\end{aligned}
$$

This procedure will be applied succesively to all the remaining rings, leading to the final positioning of the fovea, expressed by the coordinates of its center, which will be approached with a maximum error of just one pixel.

According to the above, the general expression leading to the total shift to be applied to the fovea, measured from the center of the FOV can be given by :

$$
\begin{aligned}
& \left|S t_{h}\right| \approx 2^{m}\left|S H_{m-1}\right|+2^{m-1}\left|S H_{m-2}\right|+\cdots 2\left|S H_{0}\right| \\
& \left|S t_{v}\right| \approx 2^{m}\left|S V_{m-1}\right|+2^{m-1}\left|S V_{m-2}\right|+\cdots 2\left|S V_{0}\right|
\end{aligned}
$$

The above reasoning is shown in Figs. 2.a , 2.b, and 2.c. Fig 2.a shows a lattice where the fovea is placed on the same position within the FOV as the one occupied in Fig. 1.a, but the shifts applied to fovea and ring 1 are different in both lattices : In Fig 2.a, ring 1 is shifted three rexels to the left from its center lattice position, but fovea is centered within ring 1. This fact could also be seen in Fig. 2.b, giving an unidimensional view to show the connectivity among the succesive resolution levels as well as the associated hierarchical data structure. Finally, Fig. 2.c shows the relations between the image pyramid and the asymmetric foveal polygon obtained for the lattices of Fig. 2.a.

The advantages of the geometries so obtained are : 


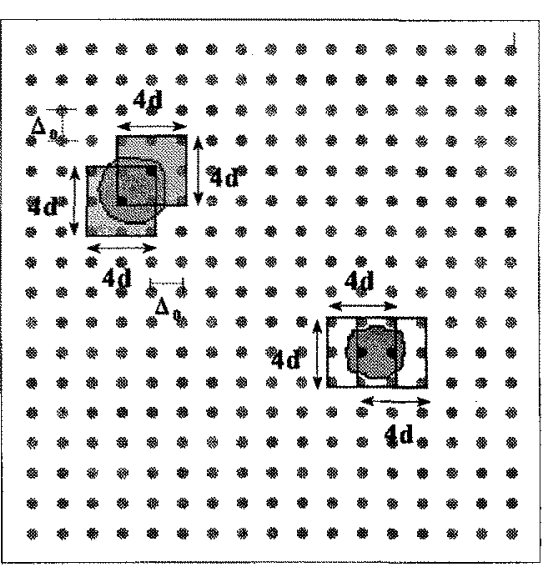

Fig 3. Possible fixation points $(\mathrm{m}=3$ and $d=8$ ) achieved with the non generalized geometries.

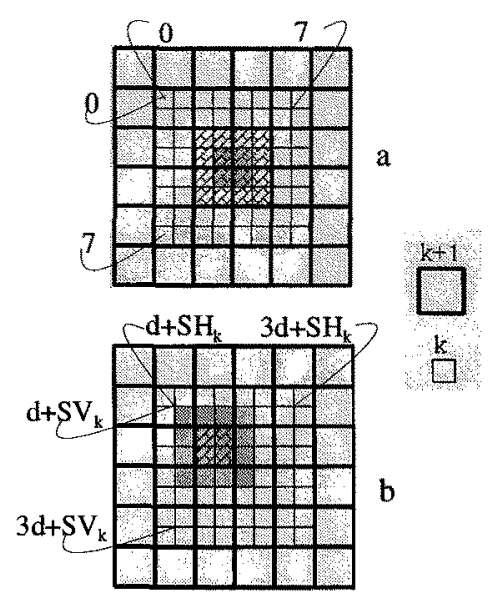

Fig 4. a) Possible father cells to a son. b) Possible son cells to a father.

1.- Fovea center can be placed onto any point of the FOV whose coordinates are even numbers, so the maximum error of placement could be one pixel, i.e. the minimum jump of 2 pixels to be applied to the fovea is independent of the configuration parameters.

$$
\Delta_{\text {forea }}=2
$$

and the minimum shift for any ring $i$ will be:

$$
\Delta_{i}=2^{i+1}
$$

2.- The number of possible fixation points is given by:

$$
N=((W-4 d) / 2)^{2}
$$

where $\mathrm{W}$ is the lateral dimension of the FOV which is given by:

$$
W=4 d \cdot 2^{m}
$$

3.- There is no possibility of having areas of the FOV unable of being examined with the highest resolution, because fovea size will always be wider than de minimum jump.

As can be seen on Fig. 3, showing allowed fixation points, for a non-generalized geometries having $m=3$ and $d=8$, when an object size is slightly bigger than $\Delta_{0}$, it could be placed in an area between two adjacent fovea positions since, because of the minimum jump separating the two foveas, it could not be possible to completely cover it with any of the foveas. Numerically, parameters $m$ and $d$ yield a $256 * 256$ scene, a $32 * 32$ pixels fovea and a minimum shift between two fixations $\Delta_{\text {fovea }}$ of 14 pixels (eq. 2).

\section{Objects detection}

By using the mechanisms given above for the shifted fovea images, whose topology and multiresolution data structure were described, objects can be detected following a procedure consisting of two phases. In the first phase, we apply a modified version of the iterative algorithm proposed by Bandera [8] for hierarchical segmentation of concentric or symmetric foveal structures. The algorithm is based on the Multiresolution Pixel Linking algorithm for pyramidal structures given by Burt and Rosenfeld [6] [7].

This first phase is performed in three steps: 
1.a) Link cells located on succesive resolution levels. Each son cell $(i, j)$ located on level $k$ will be linked to a father cell on level $k+1$. This father cell will be, among all the possible fathers cells, the one having a gray level closer to that of the son cell. The coordinates of those possible father cells are given by the expression:

$$
\begin{gathered}
\left(\lfloor(i-1) / 2\rfloor+\left(d+S V_{k}\right)+m,\right. \\
\left.\quad\lfloor(j-1) / 2\rfloor+\left(d+S H_{k}\right)+n\right) ; \quad m, n=0,1 \\
0 \leq i, j<4 d
\end{gathered}
$$

Fig. 4.a shows the possible father cells -crosshatched- suitable to be linked to the shaded son cells seen on the background.

1.b) Once the links are set, the area and gray level of father cells will be computed again according to the sons cells linked. Each father $(\mathrm{i}, \mathrm{j})$ on level $k+1$ might have a number of son cells ranging from 0 to 16. The coordinates of those potential son cells are:

$$
\begin{gathered}
\left(2\left(i-d-S V_{k}\right)+m, 2\left(j-d-S H_{k}\right)+n\right) ; \quad m, n=0,1,2,3 \\
d+S V_{k} \leq i<3 d+S V_{k} ; d+S H_{k} \leq j<3 d+S H_{k}
\end{gathered}
$$

Fig. 4.b shows the son cells -shaded-suitable to be linked to the croshatched father cells. Equations (13) and (14) can be applied to any level, from fovea -level 0- up to the waist or level containing the whole FOV. The equations are also valid for levels above the waist, with the requirement of making shiftings and subdivision factor equal to zero on all those upper levels. The linking procedure and the computation of fathers is an iterative one, finishing after stable links are obtained, i.e. links remaining unaltered after two consecutive iterations. The result is an stable data structure in which every cell on level $i$ will be linked to other cell on level $i+1$.

1.c) After obtaining this structure it is possible to generate a controllable number of segments which is a power of 4 . The procedure consists of the choice of a level $\mathrm{L}$ above the waist and of the propagation of the gray level of the cells located on that level downwards to the son cells on level L-1. That propagation will be repeated from level L-1 to level L-2, and succesively until reaching level 0 . This way, the original image will be segmented into $4^{(\mathrm{N}-\mathrm{L})}$ regions, being $\mathrm{N}$ the total number of levels within the data structure. It must be taken into account that the segments represent classes, but not neccesarily objects, since the classes may not correspond to connected regions. The second phase, namely the one to detect the objects, has three steps :

2.a) A "bottom-up" process will be performed, starting on the fovea- level 0- up to the upper levels. In this process the bounding-box coordinates (Xmin, Xmax, Ymax, Ymin) and those of the centroid $(C x, C y)$ of each cell are calculated. The bounding-boxes of cells on level $k+l$ are obtained from linked cells of level $k$. In a similar way the centroids of cells on level $k+l$ are obtained from the centroids and areas of its linked cells on level $k$.

2.b) Next, a top-down process is performed starting at a resolution level $L$ downwards to the waist. This procedure is applied to each cell in order to check if it could be labelled as the root of an object. The testing criterion is the ratio of the associated boundingbox area to the area of the cell. If the ratio is below a fixed threshold [8], the cell could be considered as a candidate to be the root of an object. To reduce the number of detections, a more restrictive test could be used by considering, besides the aforementioned ratio, the size or area of the cell, its gray level and the scene background.

2.c) The final step is to apply a corrective process to merge the roots of the potential 


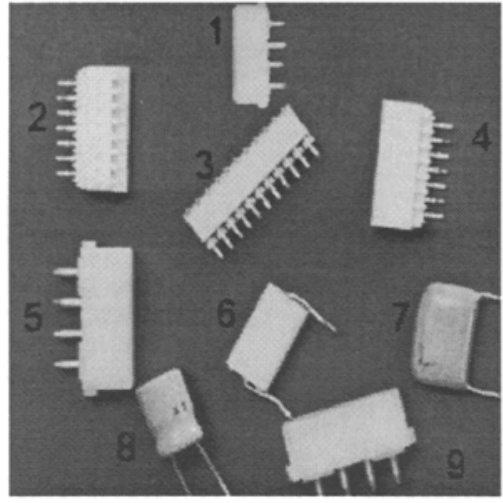

a
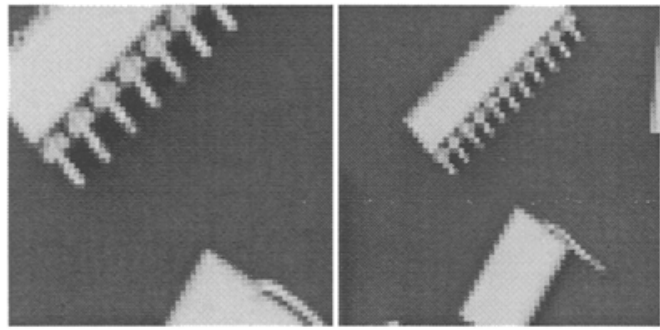

b
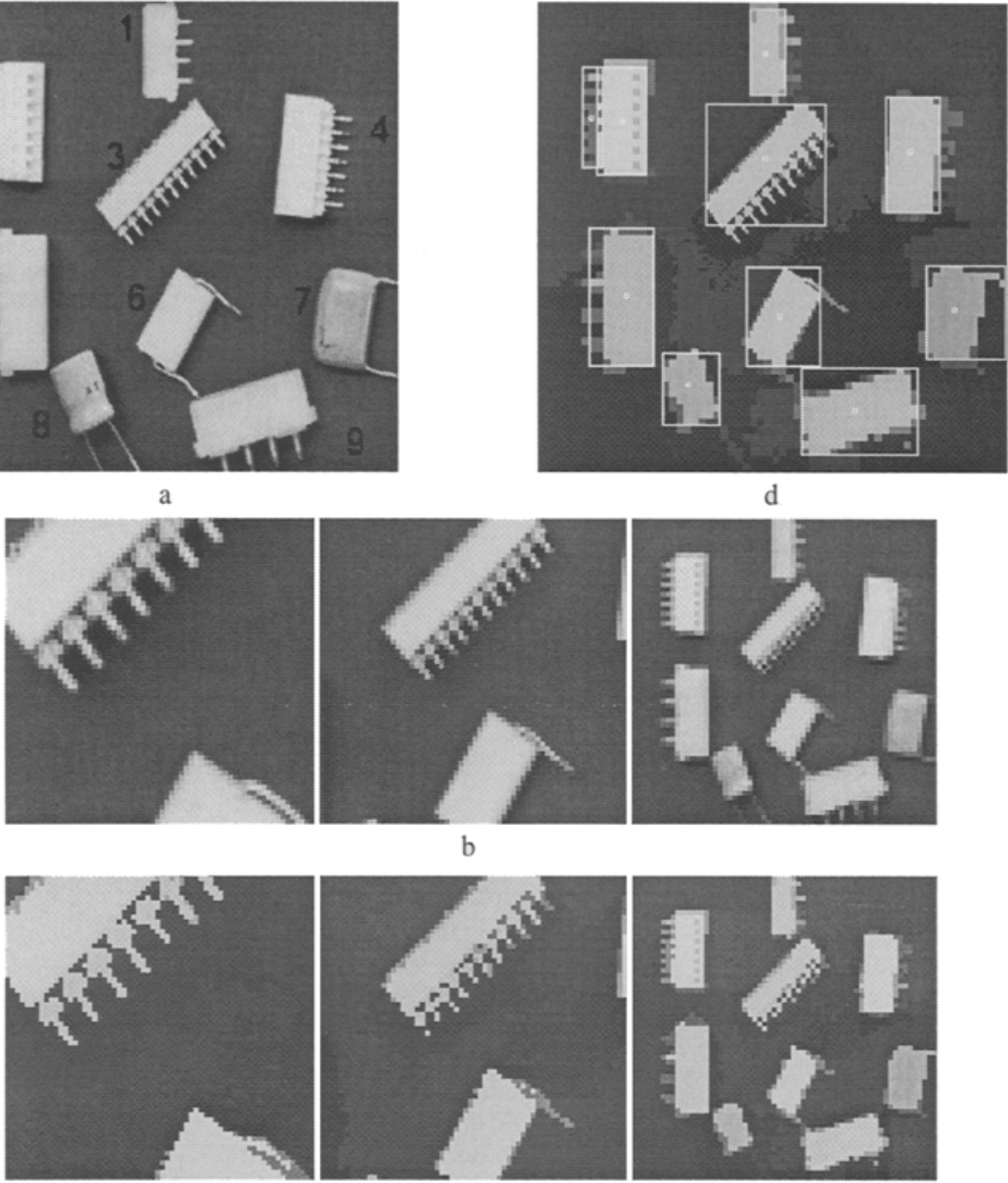

$\mathrm{d}$

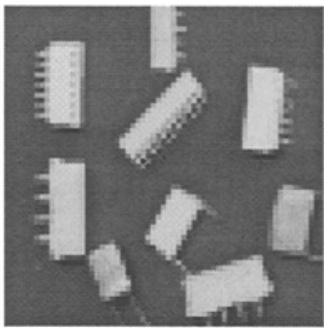

Fig 5. a) Uniform resolution image $(256 \times 256)$. b) Centered fovea levels. c) Segmented levels for centered fovea. d) Detections on the segmented foveal image. $\left(\mathrm{m}=2, \mathrm{~d}=16, \mathrm{St}_{\mathrm{h}}=\mathrm{St}_{\mathrm{v}}=0\right)$

targets detected in the previous step. This procedure is required because elongated objects are detected as a set of several objects having a certain degree of overlapping. To merge two roots, they must match, at least, three requirements: a) Their bounding-boxes must be overlapped, b) Their gray levels must be similar, and c) The roots must be connected. In this step it must be considered that bounding-boxes suitable to merge could be located on different resolution levels.

\section{Results}

Fig. 5.a shows the original image with uniform resolution, and Fig 5.b shows, the centered levels $\left(\mathrm{m}=2, \mathrm{~d}=16, \mathrm{St}_{\mathrm{h}}=\mathrm{St}_{\mathrm{v}}=0\right)$ from fovea to waist of the associated polygon. If we apply the procedures previously described to segment and compute the centroids and bounding-boxes of the potential objects, we obtain the images shown in Fig. 5.c 


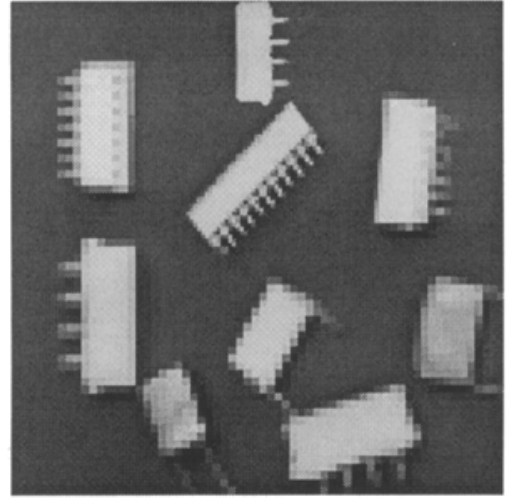

a

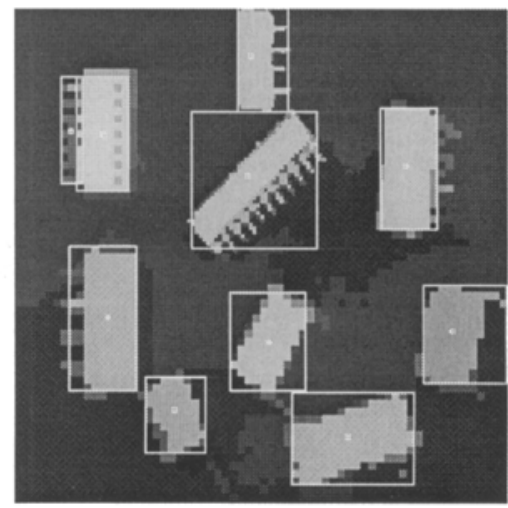

b

Fig 6. $\left(\mathrm{m}=2, \mathrm{~d}=16, \mathrm{St}_{\mathrm{h}}=0, \mathrm{St}_{\mathrm{v}}=-96\right)$

a) Foveal image. b) Detections on the segmented foveal image.

corresponding to the segmented polygon and those of Fig. 5.d indicating detections on the segmented foveal images. We can realize that all objects are detected although none of them is covered with the fovea.

If it is necessary to examine an object with the highest resolution and, at the same time, to detect whether other objects have changed their positions, the same procedures would be applied but altering the values of $\mathrm{St}_{\mathrm{h}}$ and $\mathrm{St}_{\mathrm{v}}$ given by the coordinates of the centroid of the object to examine. Namely, if we shift the fovea 96 pixels towards the upper region of the scene $\left(S t_{h}=0, S t_{v}=-96\right.$ ) we obtain the foveal image (Fig 6.a) and detections on the segmented foveal image (Fig. 6.b). It can be seen that, either placing the fovea into the center or into any other region of the scene, all objects are detected, the difference being on the choice of the region where highest resolution is desired.

It should be noticed that object number 2 is detected as two diferent objects almost overlapping each other. This is due to objet location on the peripheral region of the FOV. This is a normal result because cells on the left part of the object -the pins- have an average gray level which is very different from the one of cells on the right side -the body- (see Fig 6a). However, if the fovea is placed onto the referred object $\left(\mathrm{St}_{\mathrm{h}}=-84, \mathrm{St}_{\mathrm{v}}=-66\right)$ and the detection process is applied, there will only be one bounding-box including the whole object. This can be seen in Fig. 7 .

\section{Conclusions}

The main advantage of the shifted fovea geometries is their ability to perform object recognition on the periphery of the scene keeping a reduced data structure, because of the possibility of a selective fovea fixation on the FOV without moving the camera.

We have proposed a reconfigurable retinotopology capable of increasing the number of fovea fixation points on the scene up to the level required to reduce to one pixel the maximum fixation error. This increment of fixation points is quite important, when the objects to identify have a size similar to that of the foveal region, because object identification could be achieved at the highest resolution, irrespective of object location in the scene. In case that object dimensions are bigger than fovea dimensions, object recognition shall be done at a higher level. To achieve successful results, hierarchical 


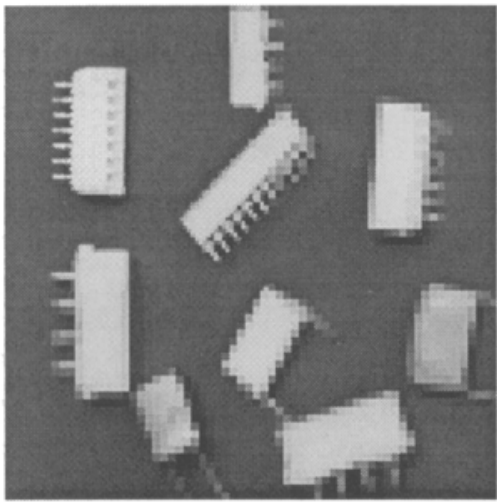

a

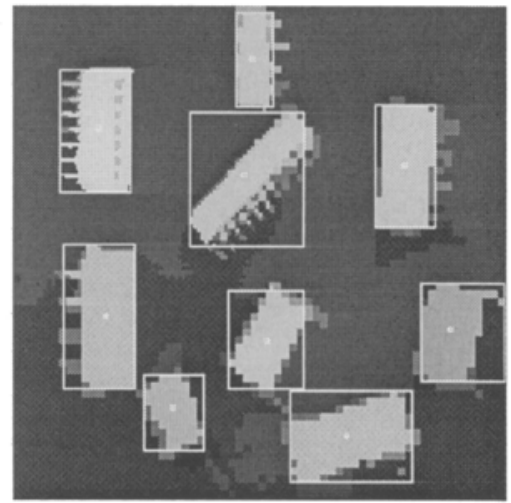

b

Fig 7. $\left(m=2, d=16, S t_{h}=-84, S t_{v}=-66\right)$

a) Foveal image .b) Detections on the segmented foveal image.

algorithms previously used were adapted to the new data structures proposed in this work to segment and detect objects found on the FOV.

\section{Acknowledgments}

The present work has been partially supported by the Spanish Comisión Interministerial de Ciencia y Tecnología, (CICYT), Proyect No. TIC095-0589.

\section{References}

[1] F. Ferrari, J. Nielsen, P. Questa and G. Sandini, Space variant imaging, Sensor Review Vol. 15, No. 2, pp. 17-20, 1995.

[2] M. J. Swain, M. A. Stricker, Promising Directions in Active Vision, NSF Active Vision Workshop, University of Chicago, August 1991.

[3] P. Camacho, F. Arrebola, F. Sandoval, Shifted Fovea Multiresolution Geometries, IEEE International Conference on Image Processing (ICIP'96), Vol. 1, pp. 307-310, Lausanne, Switzerland, 1996

[4] C. Bandera, P. Scott, Foveal Machine Vision Systems, IEEE International Conference on System, Man and Cybernetics, Cambridge, MA, Nov. 1989.

[5] P. Scott, C. Bandera, Hierarchical Multiresolution Data Structures and Algorithms for Foveal Vision Systems, IEEE International Conference on System, Man and Cybernetics, Los Angeles CA, Nov. 1990.

[6] P. J. Burt, T. H. Hong, A. Rosenfeld, Image Smoothing Based on Neighbor Linking, IEEE Trans. SMC, Vol. 11, No. 12, pp. 769-780, 1981.

[7] T. H. Hong, K. A. Narayanan, S. Peleg, A. Rosenfeld, T.Silberberg, Image Smoothing and Segmentation by Multiresolution Pixel Linking: Further Experiments and Extension, IEEE Trans. System, Man and Cyb., Vol. 12, pp. 611-622, No. 5, 1982.

[8] C. Bandera, Structures and Algortithms for Foveal Machine Vision, Amherst Systems, Tech. Report, Buffalo, NY (USA), May 1994.

[9] F. Arrebola, P. Camacho, F. Sandoval, Segmentación de Imágenes Multirresolución con Fóvea Desplazable, Actas URSI-96, Vol. 2, pp. 205-208, Madrid, España 1996. 